\title{
Matter-wave interference made clear
}

Interference patterns are generated when light from a point source passes through two parallel slits. Electrons emitted from diatomic molecules produce analogous patterns, but these couldn't be observed directly - until now.

\section{UWE BECKER}

Writing in the Proceedings of the National Academy of Sciences, ${ }^{1}$ Canton et al. report the direct observation of interference patterns in the spectra of electrons produced when diatomic molecules are irradiated with ultraviolet light. The patterns provide the first unambiguous proof that such molecules can behave as twocentre emitters of electron waves.

The question of whether light consists of particles or waves has been debated for centuries. Although Christiaan Huygens proposed in 1678 that light consists of waves, the photon was generally considered to be a particle until Thomas Young reported his classic doubleslit experiment in 1803. Young illuminated a panel containing two parallel slits with a point source of light, and observed that the light passing through the slits formed an interference pattern - a series of light and dark bands - on a screen behind the panel. This unambiguously proved the wave character of light. Imagine the confusion, then, when Arthur Compton also unambiguously proved the particulate nature of light in 1923, in studies of the scattering of high-energy photons.

Young's and Compton's contradictory results were explained by light's particlewave duality. This duality is at the heart of quantum mechanics, and is one of the prominent conceptual deviations of the field from classical physics. In fact, particle-wave duality is not limited to photons - it is a basic property of all quantum objects, including matter, as shown in 1961 by double-slit experiments involving electrons ${ }^{2}$ rather than photons.

In these experiments, an interference pattern analogous to that produced by light was observed, proving that electrons have wave properties. Since then, double-slit experiments have shown the wave character of increasingly larger quantum objects, including fullerenes $^{3}$ (buckyballs) and huge organic molecules ${ }^{4}$. Experiments to extend double-slit diffraction to truly macroscopic structures, including living organisms, are under way.

The basis of all double-slit experiments is the Heisenberg uncertainty principle, which constrains the precision with which the position and momentum of quantum objects can be measured. To obtain interference patterns, the momentum must be so precisely defined that the position of the quantum object is delocalized by more than the slit width; under these circumstances, the quantum objects are said to be coherent. If this delocalization is lost, decoherence occurs and the interference pattern disappears. Whether it is possible to determine through which slit an object passes without losing interference patterns is a long- standing question that continues to be the subject of research and controversy 5 .

In addition to uncertainty-based coherence, another mechanism can give rise to the same kind of phenomenon: coherent superposition of quantum objects emitted from spatially separated positions, often referred to as 'the molecular double-slit' (Fig. 1). An example of this occurs when homonuclear diatomic molecules, such as nitrogen $\left(\mathrm{N}_{2}\right)$, emit electrons in response to irradiation with light $^{6}$ (a process known as photoelectron emission). Electrons can be emitted coherently from both of the atoms in these molecules in such a way that the electron waves are either in phase or out of phase. These systems should therefore exhibit interference behaviour equivalent to that seen in regular double-slit experiments, an analogy first recognized 7 by Howard Cohen and Ugo Fano in 1966. They proposed a wavefunction to describe coherent emission from both atomic sites, and which predicted oscillating partial cross-sections of photoelectron emission (the cross-section is a measure of the probability that photoelectric emission will occur).

When Cohen and Fano published their findings, little evidence existed for the predicted interference oscillations - although their paper did include two graphs that plot- ted experimentally obtained crosssections against the energy of incident radiation for the valence photoionization of $\mathrm{N}_{2}$ and $\mathrm{O}_{2}$ (valence photoionization is photoelectron emission of an atom's outermost electrons). The graphs revealed obvious oscillations, which Cohen and Fano interpreted as the first hint
t. It took another 35 years before of their predicted effect. It took anothere
two-centre interference was unambiguously proven $\mathrm{H}_{2}$, and longer still to find evidence ${ }^{6}$ of it for $\mathrm{N}_{2}$.

But these reports 6,8 established the effect for the photoionization of core electrons in homonuclear diatomic molecules, rather than of valence electrons, as Cohen and Fano had reported. Furthermore, they involved techniques that did not allow direct observation of the 
interferences, necessitating a calibration of the data that introduced uncertainty into the results. Two challenges therefore remained: finding a way to directly observe interference oscillations, and obtaining proof that valence photoionization could produce interference behaviour.

Canton et al. ${ }^{1}$ have achieved both of these aims. By obtaining 'vibrationally resolved' photoionization spectra of diatomic molecules, they eliminated the calibration uncertainties that plagued the earlier core-ionization experiments. Furthermore, their approach allowed them to investigate valence photoionization for $\mathrm{N}_{2}$ and $\mathrm{H}_{2}$. The data for $\mathrm{H}_{2}$ are especially useful, because this molecule is the benchmark system modelled in most theoretical studies of photoionization.

The most unexpected result of Canton and colleagues' study is their observation of Cohen-Fano oscillations for heteronuclear diatomic molecules such as carbon monoxide (CO). In these molecules, the innermost electrons are almost completely localized at either of the two atoms, so that Cohen-Fano oscillations cannot occur. Instead, scattering of the ejected electron by the other atomic site gives rise to a different kind of oscillation, the frequency of which is twice that of the Cohen-Fano oscillation? ${ }^{\text {. }}$

But the valence orbitals of $\mathrm{CO}$ are naturally delocalized; if this delocalization is sufficiently large to cover both atoms in the molecule, then the emission of electrons could become coherent. Canton and colleagues' unusual observation of Cohen-Fano oscillations in $\mathrm{CO}$ can thus be explained by coherent valence photoionization. These findings reveal that delocalized orbitals can function as a source of two-centre interference, analogous to a Young's double-slit experiment in which the two slits have different widths.

Two-centre interference has also been observed in high-harmonic generation ${ }^{10,11}-$ a phenomenon in which molecules exposed to intense laser fields emit low-energy X-rays. Furthermore, fullerenes may be regarded as three-dimensional molecular double-slits ${ }^{12-14}$, exhibiting photoemission behaviour similar to that of one-dimensional diatomic molecules. Taking all of this together, coherent two-centre emission is emerging as an exciting subject of research with many unexpected results still to be revealed, and great potential for future applications such as quantum computing.

Uwe Becker is in the Department of Molecular Physics, FritzHaber-Institut der Max-Planck-Gesellschaft, 14195 Berlin, Germany, and in the Department of Physics, King Saud University, Riyadh, Saudi Arabia. e-mail:

beckeru@fhi-berlin.mpg.de

1. Canton, S.E. et al. Proc. Natl Acad. Sci. USA 108, 7302-7306 (2011).

2. Jönsson, C. Z. Phys. 161, 454-474 (1961).

3. Hackermüller, L., Hornberger, K., Brezger, B., Zeilinger, A. \& Arndt, M. Nature 427, 711-714 (2004)

4. Gerlich, S.et al. Nature Commun. 2, 263 (2011).

5. Kocsis, S. et al. Science 332, 1170-1173 (2011)

6. Rolles, D. et al. Nature 437, 711-715 (2005)

7. Cohen, H.D. \& Fano, U. Phys. Rev. 150, 30-33 (1966)

8. Stolterfoht, N. et al. Phys. Rev. Lett. 87, 023201 (2001).

9. Zimmermann, B. et al. Nature Phys. 4, 649-655 (2008)

10. Kanai, T., Minemoto, S. \& Sakai, H. Nature 435, 470-474 (2005).

11. Wörner, H.-J., Bertrand, J. B., Kartashov, D. V., Corkum, P. B. \& Villeneuve, D. M. Nature 466, 604-607 (2010).

12. Benning, P. J. et al. Phys. Rev. B 44, 1962-1965 (1991).

13. Xu, B., Tan, M. Q. \& Becker, U. Phys. Rev Lett. 76, 3538-3541 (1996).

14. Korica, S. et al. Surf. Sci. 604, 1940-1944 (2010). 